\title{
Task Scheduling for a Multi-robot system using Genetic Algorithm
}

\author{
Jahng Hyon Park and Jinhan Jeong
}

\begin{abstract}
In this paper, we present and solve a scheduling problem for a high-density robotic workcell under various working conditions. The genetic algorithm (GA) is employed to optimize tasks for scheduling of the multi-robot system. A new operation method for generating subsequent generations of GA, controlled mutation is introduced depending on the value of the objective function in order to help the algorithm get out of the local minimum. Several simulation graphs verify efficiency of the proposed algorithm.
\end{abstract}

Keywords- Multi-objective genetic algorithm, High-density robot, Scheduling, MTSP

\section{Introduction}

Multiple industrial robots are used for improved productivity in automobile industry. We consider a workcell where a number of robots are working simultaneously for manufacturing process of cars as shown in figure 1 . Especially, the number of points of spot welding on a single car is very large, therefore the sequence of welding by the multiple-robots affect the total tact time. The paths and sequences of the robots usually are constructed using offline teaching method. This method highly depends on not only the intuition of operator with limited information but also may take long time in a cluttered environment with high degrees of freedom robots.

In this paper, we employ the genetic algorithm with multi-objective function to optimize the paths and sequence of operations by the multiple robots.

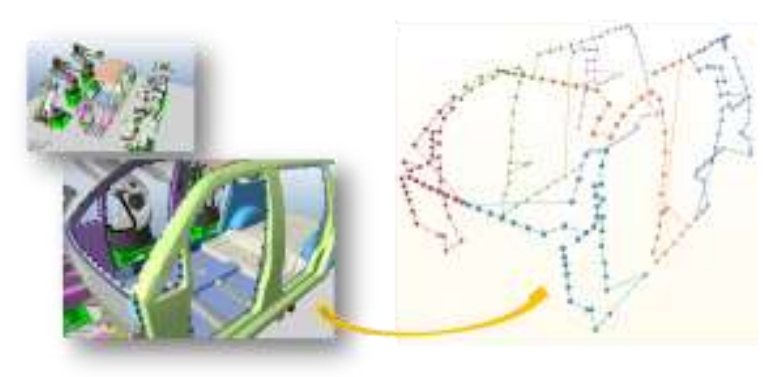

Figure 1: High density multiple robot workcell

\section{JH Park and J Jeong}

Hanyang University

Korea

\section{Genetic Algorithm}

Genetic algorithm has been successfully applied to optimization problems with large and complex search spaces due to their ability of reaching a global near optimal solution even if the search space contains multiple local minima [1]. It is usually used to find the optimal solution for single object function, on the other hand, multi-objective genetic algorithm is useful to incorporate additional objective functions.

Two types of chromosomes (Two-part chromosome representation, TCR) are employed for scheduling a robotic workcell using multi-objective genetic algorithm [2]. The first part of each chromosome is a permutation of the $n$ workcells and the second part of the chromosome is the numbers of assigned robots. The sequence of operation by each robot is tabulated as in figure 2 .

\begin{tabular}{l|c|c|c|c|c|c|c|}
\multicolumn{1}{c}{} & \multicolumn{1}{c}{$[1]$} & {$[2]$} & {$[3]$} & {$[4]$} & {$[5]$} & $\ldots$ & {$[N]$} \\
\cline { 2 - 8 } sched $_{1}$ & $p_{6}$ & $p_{3}$ & $p_{1}$ & $p_{5}$ & $p_{4}$ & $\ldots$ & $p_{4}$ \\
\cline { 2 - 8 } alloc $_{1}$ & $r_{2}$ & $r_{1}$ & $r_{3}$ & $r_{3}$ & $r_{1}$ & $\ldots$ & $r_{1}$ \\
\cline { 2 - 7 } & & & & &
\end{tabular}

Figure 2: Chromosome Encoding

The selection operator selects a chromosome from the previous population by Proportional roulette-wheel selection using the following fitness function.

$$
P(i)=F(i) / \sum F(j)
$$

where $\mathrm{P}(\mathrm{i})$ represents fitness value of the string in the population. Solutions in the current population are evaluated based on their merit to survive in the next population. This requires that each solution in a population be associated with a figure of merit or a fitness value.

Mutation changes an allele value depending on the distance of the data point to end effect of the robot. It may be recalled that each allele corresponds to a data point and its value represents the cluster to which the data point belongs [3]. The mutation operator used for the only first part of the chromosome consisted of data points.

Crossover is a recombination operator and follows the reproduction. The role of this operator is to join together parts of several individuals in order to produce new ones for the next generations [4]. According to a random selected point $k$, the crossover operator used for the first part of the chromosome, which is consisted of data points, is the order crossover. For the second part of the chromosome, the onepoint crossover is used [5]. 
The evaluation mechanism of all the chromosomes of the population is the fitness function. The value of the fitness function for one chromosome is the reflection of how well this chromosome is adapted to the environment. The Fitness function which is considered high-density robotic workcell is as follows.

$$
\mathrm{F}=\alpha \mathrm{F}+\beta \mathrm{F}+\gamma \mathrm{F}
$$

The first fitness function $F_{1}$ is to minimize the total distance of all the data points added together. This function reflects the goal of minimizing the distance required to work all $n$ points. The second fitness function $F_{2}$ is to shorten robot working time and the Third fitness function $F_{3}$ is the distance between robots and each data points. The fitness function $F$ indicates the ability of the chromosomes to survive and be reproduced in the next generation.

\section{Simulation Results}

In this section, the proposed multi-objective genetic algorithm is applied to 6 robots that have to work 338 task points in the three-dimensional space. Table 1 shows the values of the control parameters.

Table 1 Parameters used for Multi-Objective GA

\begin{tabular}{|c|c|c|c|}
\hline Setting & Value & Setting & Value \\
\hline \# of robots & 6 & $\begin{array}{l}\text { Crossover } \\
\text { Probability }\end{array}$ & $80 \%$ \\
\hline $\begin{array}{l}\# \text { of task } \\
\text { points }\end{array}$ & 338 & $\begin{array}{l}\text { MutationPro } \\
\text { bability }\end{array}$ & $8 \%$ \\
\hline $\begin{array}{l}\text { Population } \\
\text { Size }\end{array}$ & 100 & $\begin{array}{l}\text { Stagnation } \\
\text { Limit }\end{array}$ & 5000 \\
\hline
\end{tabular}

Using the genetic algorithm, the sequence of operation is optimized and the final results for each robot's operation are shown in figure 3.

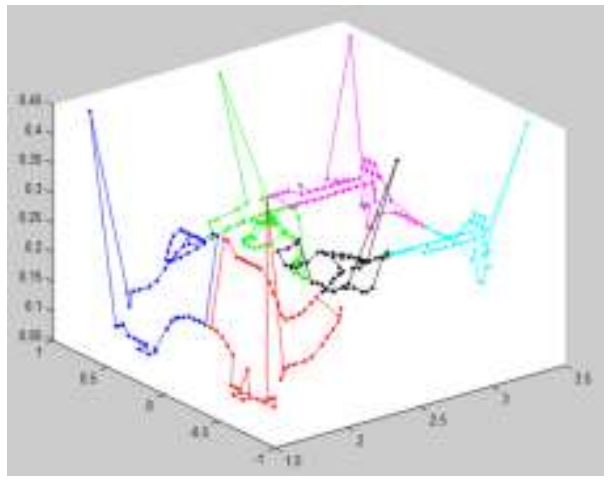

Figure 3: Optimized sequence of spot welding

The result from the tests using multi-objective GA distributes robot workcell to six parts and optimizes sequence of the end-effector's route in the three-dimensional space is shown Figure 3. Figure 4 shows fitness value convergences to the near-optimum solution.

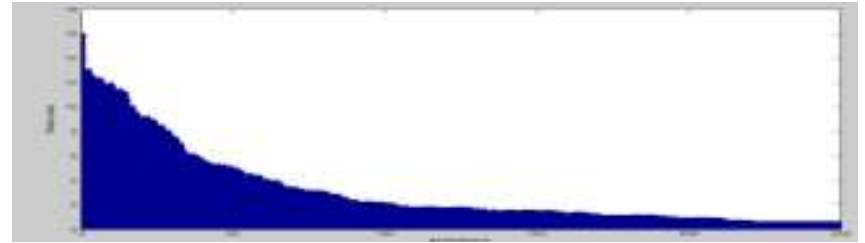

Figure 4: Fitness function

\section{Conclusions}

In this paper, we presented a multi-objective genetic algorithm for the scheduling problem which can be practical application of the high-density robotic workcell's task. Hugh-density robotic workcell is a system that aims for reducing of production area and time by arranging more and various robots in limited area. So, it needs more efficient and intelligent optimizing algorithm that requires constraint conditions for the highly-density robotic manufacturing process. Multi-objects considered various working conditions in limited area and proved to be rather fast in finding an optimum or a near-optimum solution within an affordable time.

Considering the future research work, the proposed algorithm can be added local heuristic algorithm so that it can reduce the time for optimizing a scheduling problem. In addition, to set another objective function for collisionavoidance can solve kinematic problems at the same time.

\section{References}

[1] E.K. Xidias and P.Th. Zacharia and N.A. Aspragathos, "Optimal task scheduling for a two-robot workcell," Jaurnal of systems and control engineering, vol. 224. No. 7 845-855 November 2010

[2] Yuan. S, et al. "A new approach to solving the multiple traveling salesperson problem using genetic algorithms," European Journal of Operational Research, 228(1), 72-82, 2013.

[3] K. Krishna and M. Narasimha Murty, "Genetic K-Means Algorithm," IEEE Transactions on system, Man, and Cybernetics Part B: Cybernetics, vol. 29, no. 3, June 1999.

[4] P.T. Zacharia, and N.A. Aspragathos, "Optimal robot task scheduling based on genetic algorithms,"Robotics and Computer-Integrated Manufacturing 21 67-79, 2005.

[5] Michalewitz Z, "Genetic algorithms + data structures - evolution programs," 3rd ed. Berlin: Springer; 1996. P. 21, 219-20.

[6] Tolga Bektas, "The multiple traveling salesman problem an overview of formulations and solution procedure," science direct, 34(2006) 